\title{
Plasma free amino acid profiles of Boer goat bucks as influenced by two feeding regimens
}

\author{
A.M. Almeida ${ }^{1,2 \#}$, L.M.J. Schwalbach ${ }^{2}$, H.O. de Waal $^{2}$, J.P.C. Greyling ${ }^{2}$ and L.A. Cardoso ${ }^{1}$ \\ ${ }^{1}$ IICT - Centro de Veterinária e Zootecnia and CIISA - Centro Interdisciplinar de Investigação em Sanidade Animal, \\ FMV, Rua Prof. Cid dos Santos; 1300-477 Lisboa, Portugal \\ ${ }^{2}$ Department of Animal, Wildlife \& Grassland Sciences, University of the Free State, P.O. Box 339, Bloemfontein \\ 9300, South Africa
}

\begin{abstract}
The aim of the study was to monitor the physiological changes in plasma free amino acid profiles in Boer goats under nutritional restriction. Fifteen Boer goat bucks were allocated to two experimental groups: CG (control), fed Themeda trianda hay, supplemented with maize, molasses and urea ad libitum; and RG (restricted feeding) fed the same hay ( 80 and $86 \%$ of the daily NRC energy and protein requirements, respectively) for 29 days. Animals were weighed and blood collected weekly for determination of plasma free amino acid profiles and the results compared between experimental groups. At the end of the experimental period the CG animals had higher concentrations of alanine, tyrosine and citrulline amino acids. The RG group had higher concentrations of valine, isoleucine, leucine, threonine, methionine, lysine, taurine, ornithine, hydroxyproline and tri-methyl-histidine, while glycine, serine, aspartic acid, glutamic acid, arginine, histidine and proline levels were similar in both groups. From the results it can be concluded that plasma amino acid profiles in the Boer goat were significantly affected by nutritional restriction. The degradation of short carbon chain amino acids is preferred to long carbon chain amino acids for gluconeogenesis in the physiological mechanisms of response to nutritional restriction in Boer goats.
\end{abstract}

Keywords: Boer goat, nutrition, restricted feeding, amino acids

${ }^{\#}$ Corresponding author. E-mail: aalmeida@fmv.utl.pt

\section{Introduction}

Seasonal nutritional restriction and consequent weight loss is a major characteristic of livestock production in the tropics (Collins-Lusweti, 2000). A seasonal loss of up to $40 \%$ of body weight in beef cattle and sheep can be experienced in extensive systems during the dry season with no supplementation (Jordan \& Le Fleuvre, 1989). As demonstrated by Goetsch et al. (1998), ruminant plasma free amino acid profiles seem to be linked to the nutritional status. Consequently, different plasma free amino acid profiles could be expected as a result of nutritional restriction. Under these conditions of nutritional restriction, plasma free amino acid concentrations are expected to increase as a consequence of protein breakdown in the animal to support gluconeogenesis (Bergen, 1979).

This study was aimed at defining the plasma free amino acids profiles of Boer goat bucks under nutritional restriction, hence contributing to a better understanding of the physiological responses to nutritional restrictions as part of the adaptive characteristics of this breed during periods of nutritional shortages.

\section{Materials and Methods}

Fifteen Boer goat bucks, six months old and with a mean body weight (BW) of $28 \pm 0.2 \mathrm{~kg}$ were used in this study. After two weeks of adaptation to the experimental conditions (housed in metabolism cages and fed the control diet), animals were divided into two weight-matched groups: $\mathrm{CG}$ ( $\mathrm{n}=7$; Control Group) and $\mathrm{RG}(\mathrm{n}=8$; Restricted Group). For a 28 day trial period, the $\mathrm{RG}$ animals received $500 \mathrm{~g}$ red grass (Themeda triandra) hay (92\% DM; $38 \mathrm{~g} \mathrm{CP} / \mathrm{kg} \mathrm{DM})$ per day, cut in middle August, during the local dry season, representing 80 and $86 \%$ of daily ME and CP (NRC) requirements, respectively. The CG animals received $600 \mathrm{~g}$ of red grass hay plus $170 \mathrm{~g}$ maize $(90 \% \mathrm{DM} ; 91 \mathrm{~g} \mathrm{CP} / \mathrm{kg} \mathrm{DM}), 44 \mathrm{~g}$ Calori 3000 (molasses meal; $74 \%$ $\mathrm{DM} ; 49 \mathrm{~g} \mathrm{CP} / \mathrm{kg} \mathrm{DM})$ and $15 \mathrm{~g}$ urea (99\% DM; $2857 \mathrm{~g} \mathrm{CP} / \mathrm{kg} \mathrm{DM} \mathrm{CP})$ per day, the NRC recommended maintenance nutritional level. Blood samples were collected and body weight was determined weekly. One 
hour prior to feeding in the morning blood was collected by venipuncture (vena jugularis) into heparinized vacutainer ${ }^{\circledR}$ tubes and centrifuged at $200 \mathrm{G}$ for 15 minutes. Plasma was separated and placed in tubes and stored at $-20{ }^{\circ} \mathrm{C}$ for further analysis. Plasma $(300 \mu \mathrm{L})$ was de-proteinised with sulphosalicilic acid, centrifuged at $450 \mathrm{G}$ for 15 minutes and subjected to $\mathrm{pH}$ reduction to 2.2 using lithium hydroxide. Free amino acids were assayed using a HPLC and Beckman Coulter Amino Acid Analyser 7300 (Fullerton, CA, USA) in a lithium buffer system.

Plasma amino acid profiles were compared by ANOVA-repeated measures analyses (SAS, 1990). Data are presented as the mean \pm s.e.m.

\section{Results and Discussion}

During the trial period, on average, animals in the CG group increased by $3.8 \mathrm{~kg}$ in BW, while those in the RG group lost $5.6 \mathrm{~kg}$ (a $10 \%$ increase and a $20 \%$ decrease in $\mathrm{BW}$, respectively relative to the onset of the experiment) as shown in Figure 1.

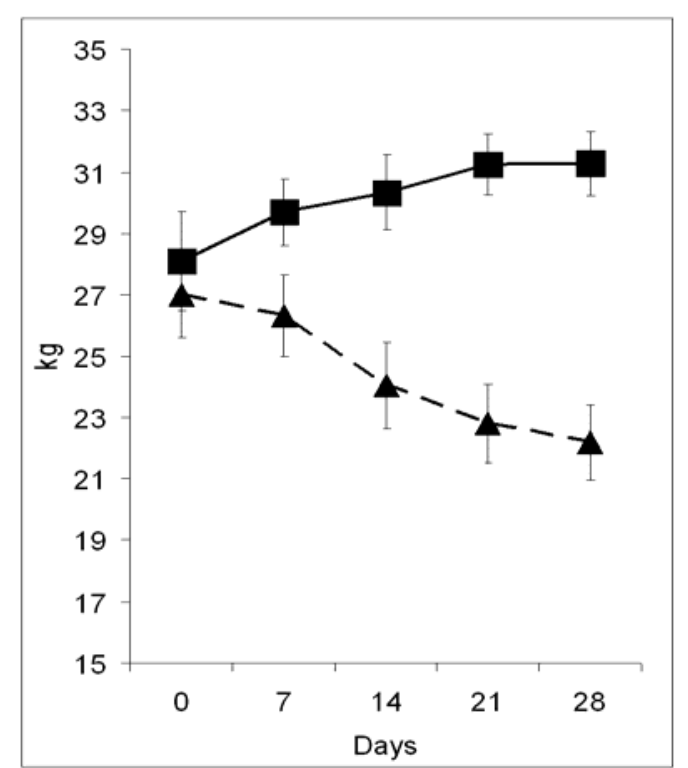

Figure 1 Live weight $(\mathrm{kg})$ changes in control CG $(\boldsymbol{\bullet})$ and restricted RG $(\boldsymbol{\Delta})$ fed Boer goat bucks. Error bars indicate s.e.m.

Plasma amino acid concentrations are shown in Table 1. At the end of the trial, differences $(\mathrm{P}<0.05)$ between the $\mathrm{CG}$ and $\mathrm{RG}$ groups were observed for the following amino acids: alanine (Ala), tyrosine (Tyr) and citrulline (Cit) (21, 32 and 50\% respectively higher plasma concentrations than for the CG group) and valine (Val), leucine (Leu), isoleucine (Ile), threonine (Thr), methionine (Met), lysine (Lys), taurine (Tau), ornithine (Orn), hydroxyproline (Hyp) and tri-methyl histidine (Me3His) (52, 57, 30, 29, 61, 91, 41, 55, 300 and $560 \%$ higher concentrations in the RG group, compared to the CG group on day 28 of the trial). The plasma concentrations of glycine (Gly), serine (Ser), aspartic acid (Asp), glutamic acid (Glu), arginine (Arg), histidine (His) and proline (Pro) were generally similar for both groups at this stage.

The results obtained in this study indicated the particular increase in plasma free amino acid concentrations to be associated with the degradation of the succinyl CoA and acetoacetyl CoA pathways. The amino acids generally degraded by the $\alpha$-ketoglutarate, pyruvate and oxaloacetate pathways, maintained similar concentrations in both experimental groups. Ala, Glu, Ser, Asp and Gly are the most important gluconeogenic amino acids, contributing 8 to $9 \%$ of the total glucose produced for maintenance in sheep (the equivalent to all other remaining amino acids) under restricted nutrition, as demonstrated by Wolff \& Bergman (1972).

In this study the amino acids, Ala, Glu, Ser, Asp and Gly, maintained similar levels throughout the experimental period in the RG animals, with no significant differences in the CG animals. These results could have been caused by a faster degradation of these amino acids that are probably used as precursors for 
gluconeogenesis, which prevented their accumulation in blood plasma - unlike Val, Leu, Ile, Thr, Met, Lys and Ile and His that accumulated. In situations of severe nutritional restriction, amino acids with a higher number of C-atoms (Val, Leu, Ile, Thr, Met, Lys and His) tended to exhibit increased levels in the plasma, while amino acids with less $\mathrm{C}$-atoms showed similar concentrations to those observed at the onset of the nutritional restriction period and in the CG animals.

Table 1 Mean ( \pm s.e.m.) plasma free amino acid profiles $(\mu \mathrm{mol} / \mathrm{L})$ in the control $(\mathrm{CG})$ and nutritionally restricted (RG) Boer goat bucks during a 28 day feeding trial

\begin{tabular}{|c|c|c|c|c|c|c|c|c|}
\hline \multirow[b]{2}{*}{$\begin{array}{l}\text { Amino } \\
\text { acid }\end{array}$} & \multicolumn{2}{|c|}{ Day 7} & \multicolumn{2}{|c|}{ Day 14} & \multicolumn{2}{|c|}{ Day 21} & \multicolumn{2}{|c|}{ Day 28} \\
\hline & $\begin{array}{c}\mathrm{CG} \\
(\mathrm{n}=7)\end{array}$ & $\begin{array}{c}\mathrm{RG} \\
(\mathrm{n}=8)\end{array}$ & $\begin{array}{c}\mathrm{CG} \\
(\mathrm{n}=7)\end{array}$ & $\begin{array}{c}\mathrm{RG} \\
(\mathrm{n}=8)\end{array}$ & $\begin{array}{c}\mathrm{CG} \\
(\mathrm{n}=7)\end{array}$ & $\begin{array}{c}\mathrm{RG} \\
(\mathrm{n}=8)\end{array}$ & $\begin{array}{c}\mathrm{CG} \\
(\mathrm{n}=7)\end{array}$ & $\begin{array}{c}\mathrm{RG} \\
(\mathrm{n}=8)\end{array}$ \\
\hline Gly & $1465^{\mathrm{a}}$ & $1368^{\text {ab }}$ & $\begin{array}{c}990^{\mathrm{c}} \\
(547)\end{array}$ & $\begin{array}{l}1135^{b} \\
(7999\end{array}$ & $1454^{\mathrm{a}}$ & $1130^{b}$ & $\begin{array}{l}1128^{b} \\
(6544)\end{array}$ & $\begin{array}{l}1030^{c} \\
(526)\end{array}$ \\
\hline Ala & $\begin{array}{l}(86.1) \\
288^{\mathrm{a}}\end{array}$ & $\begin{array}{l}(94.3) \\
215^{\mathrm{b}}\end{array}$ & $199^{\mathrm{b}}$ & $198^{\mathrm{b}}$ & $276^{\mathrm{a}}$ & $195^{\mathrm{b}}$ & $230^{\mathrm{ab}}$ & $190^{\mathrm{b}}$ \\
\hline & $(22.7)$ & (11.7) & (14.1) & (14.8) & $(23.0)$ & (16.2) & $(12.3)$ & (13.2) \\
\hline Val & $\begin{array}{c}234^{\mathrm{b}} \\
(13.6)\end{array}$ & $\begin{array}{l}269^{\mathrm{ab}} \\
(9.5)\end{array}$ & $\begin{array}{l}184^{\mathrm{c}} \\
(14.3)\end{array}$ & $\begin{array}{l}280^{\mathrm{ab}} \\
(12.8)\end{array}$ & $\begin{array}{l}211^{\text {bc }} \\
(8.7)\end{array}$ & $\begin{array}{l}275^{\mathrm{ab}} \\
(17.5)\end{array}$ & $\begin{array}{l}194^{\mathrm{bc}} \\
(12.3)\end{array}$ & $\begin{array}{c}295^{\mathrm{a}} \\
(23.8)\end{array}$ \\
\hline Leu & $\begin{array}{l}137^{b} \\
(9.7)\end{array}$ & $\begin{array}{l}157^{\mathrm{ab}} \\
(7.3)\end{array}$ & $\begin{array}{c}104^{\mathrm{c}} \\
(10.7)\end{array}$ & $\begin{array}{c}172^{\mathrm{a}} \\
(10.8)\end{array}$ & $\begin{array}{l}111^{\mathrm{bc}} \\
(6.7)\end{array}$ & $\begin{array}{c}147^{\mathrm{ab}} \\
(11.6)\end{array}$ & $\begin{array}{l}102^{\mathrm{bc}} \\
(9.6)\end{array}$ & $\begin{array}{c}160^{\mathrm{ab}} \\
(11.8)\end{array}$ \\
\hline Ile & $65.7^{b}$ & $82.3^{\mathrm{a}}$ & $57.7^{b}$ & $93.5^{\mathrm{a}}$ & $56.9^{b}$ & $\begin{array}{l}74.0^{\mathrm{ab}} \\
(4.4)\end{array}$ & $56.4^{\mathrm{b}}$ & $\begin{array}{c}72.9^{\mathrm{ab}} \\
(44)^{2}\end{array}$ \\
\hline Ser & $\begin{array}{l}86.0^{\mathrm{a}} \\
(7.1)\end{array}$ & $\begin{array}{l}85.6^{\mathrm{a}} \\
(4.9)\end{array}$ & $\begin{array}{l}63.6^{\mathrm{ab}} \\
(9.3)\end{array}$ & $\begin{array}{l}81.5^{\mathrm{a}} \\
(5.7)\end{array}$ & $\begin{array}{l}75.9^{\mathrm{a}} \\
(6.8)\end{array}$ & $\begin{array}{l}74.5^{\mathrm{a}} \\
(7.0)\end{array}$ & $\begin{array}{c}63.7^{\mathrm{ab}} \\
(5.6)\end{array}$ & $\begin{array}{r}79.3^{\mathrm{a}} \\
(20.4)\end{array}$ \\
\hline Thr & $\begin{array}{l}74.9^{b c} \\
(11.8)\end{array}$ & $\begin{array}{c}98.8^{\mathrm{ab}} \\
(1.9)\end{array}$ & $\begin{array}{l}41.3^{\mathrm{d}} \\
(10.6)\end{array}$ & $\begin{array}{c}105.9^{\mathrm{a}} \\
(8.6)\end{array}$ & $\begin{array}{l}62.0^{\mathrm{cd}} \\
(7.2)\end{array}$ & $\begin{array}{c}113.4^{\mathrm{a}} \\
(8.2)\end{array}$ & $\begin{array}{c}51.3^{\text {cd }} \\
(9.0)\end{array}$ & $\begin{array}{l}116.9^{\mathrm{a}} \\
(12.8)\end{array}$ \\
\hline Met & $\begin{array}{c}11.6^{\mathrm{bc}} \\
(2.4)\end{array}$ & $\begin{array}{l}18.9^{\mathrm{a}} \\
(1.3)\end{array}$ & $\begin{array}{c}11.7^{\text {bc }} \\
(2.1)\end{array}$ & $\begin{array}{l}23.6^{\mathrm{a}} \\
(2.4)\end{array}$ & $\begin{array}{l}11.1^{\mathrm{c}} \\
(2.0)\end{array}$ & $\begin{array}{l}22.0^{\mathrm{a}} \\
(2.5)\end{array}$ & $\begin{array}{l}9.1^{\mathrm{c}} \\
(2.0)\end{array}$ & $\begin{array}{l}23.5^{\mathrm{a}} \\
(5.3)\end{array}$ \\
\hline Asp & $\begin{array}{l}17.6^{\mathrm{a}} \\
(0.9)\end{array}$ & $\begin{array}{c}16.0^{\mathrm{ab}} \\
(0.9)\end{array}$ & $\begin{array}{l}17.1^{\mathrm{ab}} \\
(1.2)\end{array}$ & $\begin{array}{l}14.3^{\text {bc }} \\
(1.3)\end{array}$ & $\begin{array}{l}18.7^{\mathrm{a}} \\
(1.0)\end{array}$ & $\begin{array}{c}14.9^{\mathrm{ab}} \\
(1.4\end{array}$ & $\begin{array}{l}17.0^{\mathrm{ab}} \\
(1.3)\end{array}$ & $\begin{array}{l}12.6^{\mathrm{C}} \\
(1.7)\end{array}$ \\
\hline Glu & $\begin{array}{c}101^{\mathrm{ab}} \\
(9.2)\end{array}$ & $\begin{array}{c}105.3^{\mathrm{ab}} \\
(7.3)\end{array}$ & $\begin{array}{l}111^{\mathrm{a}} \\
(8.9)\end{array}$ & $\begin{array}{c}105.4^{\mathrm{ab}} \\
(10.7)\end{array}$ & $\begin{array}{l}75.0^{\mathrm{b}} \\
(5.8)\end{array}$ & $\begin{array}{c}86.4^{\mathrm{ab}} \\
(6.8)\end{array}$ & $\begin{array}{c}74.5^{\mathrm{b}} \\
(12.8)\end{array}$ & $\begin{array}{c}92.1^{\mathrm{ab}} \\
(7.8)\end{array}$ \\
\hline Lys & $\begin{array}{l}136.7^{\mathrm{b}} \\
(21.7)\end{array}$ & $\begin{array}{l}202.8^{\mathrm{a}} \\
(11.7)\end{array}$ & $\begin{array}{c}76.3^{\mathrm{c}} \\
(12.7)\end{array}$ & $\begin{array}{l}184.9^{\mathrm{a}} \\
(12.2)\end{array}$ & $\begin{array}{c}97.3^{\mathrm{bc}} \\
(2.7)\end{array}$ & $\begin{array}{l}207.3^{\mathrm{a}} \\
(15.7)\end{array}$ & $\begin{array}{l}108^{\mathrm{bc}} \\
(8.6)\end{array}$ & $\begin{array}{l}205.9^{\mathrm{a}} \\
(17.9)\end{array}$ \\
\hline Arg & $\begin{array}{l}92.3^{\mathrm{ab}} \\
(19.7)\end{array}$ & $\begin{array}{c}103.0^{\mathrm{a}} \\
(9.7)\end{array}$ & $\begin{array}{l}81.7^{\mathrm{ab}} \\
(12.5)\end{array}$ & $\begin{array}{c}90.5^{\mathrm{ab}} \\
(7.4)\end{array}$ & $\begin{array}{l}86.7^{\mathrm{ab}} \\
(10.3)\end{array}$ & $\begin{array}{l}104.6^{\mathrm{a}} \\
(11.7)\end{array}$ & $\begin{array}{c}97.3^{\mathrm{a}} \\
(13.3)\end{array}$ & $\begin{array}{l}104.4^{\mathrm{a}} \\
(16.9)\end{array}$ \\
\hline His & $\begin{array}{c}56.6^{\mathrm{ab}} \\
(5.6)\end{array}$ & $\begin{array}{c}64.5^{\mathrm{ab}} \\
(2.5)\end{array}$ & $\begin{array}{c}42.7^{\mathrm{bc}} \\
(4.1)\end{array}$ & $\begin{array}{l}67.0^{\mathrm{a}} \\
(4.0)\end{array}$ & $\begin{array}{l}52.3^{\mathrm{b}} \\
(5.0)\end{array}$ & $\begin{array}{c}62.4^{\mathrm{ab}} \\
(2.9)\end{array}$ & $\begin{array}{c}44.6^{\mathrm{bc}} \\
(2.4)\end{array}$ & $\begin{array}{c}58.8^{\mathrm{a} b} \\
(7.5)\end{array}$ \\
\hline Tyr & $\begin{array}{c}26.1^{\mathrm{ab}} \\
(3.2)\end{array}$ & $\begin{array}{l}13.6^{\mathrm{b}} \\
(2.2)\end{array}$ & $\begin{array}{l}37.1^{\mathrm{a}} \\
(6.0)\end{array}$ & $\begin{array}{l}15.9^{b} \\
(2.2)\end{array}$ & $\begin{array}{l}40.7^{\mathrm{a}} \\
(5.5)\end{array}$ & $\begin{array}{l}15^{\mathrm{b}} \\
(1.4)\end{array}$ & $\begin{array}{l}34.3^{\mathrm{a}} \\
(4.9)\end{array}$ & $\begin{array}{l}14.8^{\mathrm{b}} \\
(3.6)\end{array}$ \\
\hline Pro & $\begin{array}{l}198^{\mathrm{a}} \\
(9.9)\end{array}$ & $\begin{array}{l}182^{\mathrm{ab}} \\
(4.7)\end{array}$ & $\begin{array}{l}135^{\mathrm{c}} \\
(8.0)\end{array}$ & $\begin{array}{c}167^{\mathrm{b}} \\
(10.2)\end{array}$ & $\begin{array}{l}179^{\mathrm{ab}} \\
(8.8)\end{array}$ & $\begin{array}{c}185^{\mathrm{ab}} \\
(10.9)\end{array}$ & $\begin{array}{l}150^{\mathrm{bc}} \\
(9.3)\end{array}$ & $\begin{array}{c}189^{\mathrm{ab}} \\
(12.3)\end{array}$ \\
\hline Tau & $\begin{array}{l}93.6^{b c} \\
(19.7)\end{array}$ & $\begin{array}{l}61.9^{b c} \\
(12.1)\end{array}$ & $\begin{array}{c}52.7^{\mathrm{bc}} \\
(9.1)\end{array}$ & $\begin{array}{c}100^{\mathrm{b}} \\
(15.3)\end{array}$ & $\begin{array}{l}66.3^{b c} \\
(18.0)\end{array}$ & $\begin{array}{l}162.8^{\mathrm{a}} \\
(18.3)\end{array}$ & $\begin{array}{l}57.3^{b c} \\
(11.6)\end{array}$ & $\begin{array}{l}195.6^{\mathrm{a}} \\
(33.0)\end{array}$ \\
\hline Orn & $\begin{array}{l}69.6^{\mathrm{bc}} \\
(16.8)\end{array}$ & $\begin{array}{c}81.9^{\mathrm{bc}} \\
(5.3)\end{array}$ & $\begin{array}{l}59.9^{\mathrm{c}} \\
(4.8)\end{array}$ & $\begin{array}{l}91.9^{\mathrm{b}} \\
(8.6)\end{array}$ & $\begin{array}{l}64.9^{\mathrm{c}} \\
(4.7)\end{array}$ & $\begin{array}{c}101.6^{\mathrm{ab}} \\
(7.6)\end{array}$ & $\begin{array}{l}76.7^{\text {bc }} \\
(8.05)\end{array}$ & $\begin{array}{c}119^{\mathrm{a}} \\
(11.6)\end{array}$ \\
\hline Cit & $\begin{array}{l}85.3^{\mathrm{ab}} \\
(12.0)\end{array}$ & $\begin{array}{c}56.9^{b c} \\
(8.4)\end{array}$ & $\begin{array}{c}106^{\mathrm{a}} \\
(10.4)\end{array}$ & $\begin{array}{l}52.1^{\mathrm{bc}} \\
(5.7)\end{array}$ & $\begin{array}{l}86^{\mathrm{ab}} \\
(6.7)\end{array}$ & $\begin{array}{l}75.1^{b} \\
(6.5)\end{array}$ & $\begin{array}{c}105^{\mathrm{a}} \\
(12.8)\end{array}$ & $\begin{array}{c}70^{\mathrm{b}} \\
(9.1)\end{array}$ \\
\hline HyP & $\begin{array}{c}44.7^{\mathrm{bc}} \\
(5.0)\end{array}$ & $\begin{array}{c}43.8^{\mathrm{bc}} \\
(3.9)\end{array}$ & $\begin{array}{l}34.7^{\mathrm{c}} \\
(5.3)\end{array}$ & $\begin{array}{l}54.0^{\mathrm{b}} \\
(7.4)\end{array}$ & $\begin{array}{l}28.7^{\mathrm{c}} \\
(2.9)\end{array}$ & $\begin{array}{l}76.6^{\mathrm{a}} \\
(9.4)\end{array}$ & $\begin{array}{l}25.4^{\mathrm{c}} \\
(2.7)\end{array}$ & $\begin{array}{l}75.3^{\mathrm{a}} \\
(6.9)\end{array}$ \\
\hline Me3His & $\begin{array}{l}65.6^{\mathrm{d}} \\
(6.5)\end{array}$ & $\begin{array}{l}91.9^{c} \\
(5.8)\end{array}$ & $\begin{array}{c}49.0^{\mathrm{de}} \\
(7.6)\end{array}$ & $\begin{array}{c}125.4^{\mathrm{b}} \\
(6.9)\end{array}$ & $\begin{array}{c}48.4^{\mathrm{de}} \\
(6.7)\end{array}$ & $\begin{array}{c}159.9^{\mathrm{a}} \\
(9.0)\end{array}$ & $\begin{array}{l}30.9^{\mathrm{e}} \\
(4.5)\end{array}$ & $\begin{array}{l}175.0^{\mathrm{a}} \\
(16.9)\end{array}$ \\
\hline
\end{tabular}

${ }_{\mathrm{a}, \mathrm{b}, \mathrm{c}, \mathrm{d}}$ Values with different superscripts within the same row differ significantly $(\mathrm{P}<0.05)$. Gly - glycine; Ala - alanine; Val - valine; Leu - leucine; Ile - isoleucine; Ser - serine; Thr - threonine; Met - methionine; Asp - aspartate; Glu glutamic acid; Lys - lysine; Arg - arginine; His - histidine; Tyr - tyrosine; Pro - proline; Tau - taurine; Orn ornithine; Cit - citrulline; HyP - hydroxyproline and Me3His - tri-methyl-histidine 
The catabolism of branched-chain amino acids was lower due to the enzyme BCOADH (branched chain oxo-acid dehydrogenase) which is much less important in ruminants than in monogastric animals (Lobley, 1992). This possibly explains the higher concentration of leucine in nutritionally restricted animals. Branched-chain amino acids (derived from muscle protein catabolism) would therefore tend to accumulate in the blood, due to a lower activity of BCOADH in ruminants.

Regarding the amino acids of the urea cycle (Arg, Cit and Orn), CG animals had higher plasma concentrations of Cit and lower plasma concentrations of Orn by comparison. Similar plasma concentrations of Arg were recorded in both groups, possibly since this amino acid results from the degradation of Asp through the formation of arginino-succinate (Umbarger \& Zubay, 1988). No significant difference was registered for the latter amino acid. Under conditions of nutritional restriction urea excretion tends to diminish as a nitrogen savings mechanism. This could justify the increase in Orn concentration as a result of restricted feeding as this amino acid is also a product of Arg degradation (Umbarger \& Zubay, 1988). A lower plasma Cit concentration in the RG animals may be due to the reduction of the concentrations of carbomoxyl phosphate, caused by a decrease of $\mathrm{NH}_{3}$ availability.

The restricted fed animals had higher plasma concentrations of 3-methyl histidine, possibly as a result of the serious nutritional restrictions to which they were subjected. This indicates a high level of myofibrillar protein degradation and amino acid de-amination for gluconeogenesis. The physiological responses to energy restrictions result in the degradation of body proteins, including those of myofibrillar origin for gluconeogenesis. This from a physiological viewpoint is an expensive way to liberate energy to maintain homeostasis and the basal metabolism. Producers should be made aware of this fact and try to avoid severe nutritional restrictions of their animals by strategic supplementation during periods of feed scarcity.

\section{Acknowledgements}

The authors would like to thank Fundação para a Ciência e a Tecnologia (PRAXIS XXI/BM/17921/98) for financial support, and T. Müller, D. du Bruÿn and W. Combrink (University of the Free State) for their valuable assistance.

\section{References}

Bergen, W.G., 1979. Free amino acids in blood of ruminants - Physiological and nutritional regulation. J. Anim. Sci. 49, 1577-1589.

Collins-Lusweti, E., 2000. The performance of the Nguni, Afrikander and Bonsmara cattle breeds in developing areas of Southern Africa. S. Afr. J. Anim. Sci. 30, 28-29.

Goetsch, A.L., Galloway, D.L. \& Patil, A.R., 1998. Arterial amino acid concentrations in sheep consuming forage diets. Small Rumin. Res. 29, 51-60.

Jordan, D.J. \& Le Feuvre, A.S., 1989. The extent and cause of perinatal lamb mortality in three flocks of Merino sheep. Aust. Vet. J. 66 198-201.

Lobley, G.E., 1992. Control of the metabolic fate of amino acids in ruminants: A review. J. Anim. Sci., 70 3264-3275.

SAS, 1990. SAS User's Guide, Version 6, $4^{\text {th }}$ ed. SAS Institute, Inc., Cary, N.C., USA.

Umbarger, H.E. \& Zubay, G., 1988. Catabolism of amino acids. In: Biochemistry. Eds. Zubay G., Macmillan, New York. pp. 615-643.

Wolff, J.E. \& Bergman, E.N., 1972. Gluconeogenesis from plasma amino acids in fed sheep. Am. J. Physiol. $223,455-460$. 